Laurent Bourdillon

Christine Wandrey

\section{On-line study of polyelectrolyte network formation by interfacial reaction}

Received: 12 December 2003

Accepted: 9 February 2004

Published online: 1 April 2004

(C) Springer-Verlag 2004

L. Bourdillon · C. Wandrey

Institute of Chemical and Biological

Process Science, Swiss Federal Institute of Technology, 1015 Lausanne, Switzerland

C. Wandrey $(\square)$

EPFL-ISP-LBCh,

1015 Lausanne, Switzerland

E-mail: christine.wandrey@epfl.ch

Tel.: + 41-21-6933672

Fax: +41-21-6936030

\begin{abstract}
A modified synthetic boundary experiment of analytical ultracentrifugation has been employed to examine, on-line, polyelectrolyte complex formation at flat interfaces yielding highly swollen membranes/networks. Systematic experiments with sodium alginate as a polyanion and chitosan and poly(L-lysine) as polycations identified the influence of concentration, $\mathrm{pH}$, molar mass, and polycation type on the membrane characteristics and the formation process. The
\end{abstract}

membranes have been evaluated by five characteristics defined herein: total thickness, compactness, heterogeneity, symmetry, and growth. The results confirm the sensitivity of the method suited to elaborate general relationships for polyelectrolyte membrane design.

Keywords Polyelectrolyte complex · Analytical ultracentrifugation Sodium alginate $\cdot$ Chitosan . Poly(L-lysine)

\section{Introduction}

The solubility of charged macromolecules, polyelectrolytes, in polar solvents is primarily based on the presence of dissociating ionic groups [1]. Mixing solutions of oppositely charged macromolecules, polyanions (PAs) and polycations (PCs), spontaneously yields polyelectrolyte complexes [2]. During this complex formation complete or partial charge neutralization occurs, leading, in general, to phase separation and/or nonspecific precipitation [3,4]. The combination of a limited number of PA/PC pairs results in highly swollen network structures exhibiting membrane properties if the complex formation takes place at well-defined interfaces. These can be of any shape, for example, flat or spherical, as long as the macromolecules are allowed to interpenetrate by self-diffusion and the polyion concentration is sufficiently high.

Such semipermeable materials have become increasingly interesting as materials for isolation, protection, storage, or delivery purposes in areas such as cosmetics, personal care, pharmacy, biotechnology, and medicine
[5]. The specific applications require the design of different network properties, including mechanical stability, durability, and permeability. Moreover, in order to select the right technology and formation process conditions, knowledge about the formation kinetics is advantageous. However, the prediction of polyelectrolyte complex networks/membranes, as a function of macromolecular, physicochemical, structural, and environmental conditions, has not been achieved to date.

Recently, the synthetic boundary experiment and the optical system of an analytical ultracentrifuge (AUC) have been proposed to study the membrane formation, on-line, at a flat interface [6]. Both the experimental principle and the sensitivity for various component combinations have been demonstrated by screening experiments [7].

Analytical ultracentrifugation is well established for the characterization of macromolecules, colloids, or nanoparticles by a variety of parameters [8]. Since a new generation of equipment became available, which allows fast on-line data acquisition, and partially evaluation, 
the basic techniques of analytical ultracentrifugation have been employed to study other materials and even reactions $[9,10,11]$. One of these techniques is the synthetic boundary experiment, where two liquids come into contact at a well-defined surface. The appropriate synthetic boundary cell has been selected to study the polyelectrolyte complex formation [6].

Systematic experiments have been performed to identify the influence of the polyion concentration and the solution $\mathrm{pH}$ in the case of the two-component polyelectrolyte network alginate/chitosan (Ch). Additional studies with poly(L-lysine) (PLL) as a PC reacting with sodium alginate (SA) $/ \mathrm{CaCl}_{2}$ served to evaluate the influence of the molar mass. The $\mathrm{pH}$ has a minor influence on the charge density at the polymer chain of SA but determines the degree of protonation at the $\mathrm{Ch}$ and PLL chains. However, in order to progress and, finally, conclude interdependencies between chemical structure, macromolecular characteristics, and experimental conditions, membrane evaluation parameters had to be defined. Five are reported here, including their application to the networks/membranes formed during the two selected experimental series.

\section{Materials and methods}

\section{Materials}

SA (Keltone HV, Kelco Chemical Company, San Diego, USA, batch no. 54660) was used as a PA for all the experiments. It was characterized by an intrinsic viscosity $[\eta]=807 \mathrm{ml} / \mathrm{g}$ in $0.1 \mathrm{M} \mathrm{NaCl}$, partial specific volumes $\bar{v}=400 \mathrm{ml} / \mathrm{g}$ in water and $\bar{v}=0.470 \mathrm{ml} / \mathrm{g}$ in $0.1 \mathrm{~m} \mathrm{NaCl}$, and an $\alpha$-L-guluronic acid structure content (G content) of $40 \%$ determined by ${ }^{1} \mathrm{H}$ NMR [12].

Various PCs were employed for the complex formation. Modified oligochitosan was prepared as described previously [13]. PLL hydrobromide samples were purchased from Sigma (Switzerland). The characteristics of all the polymeric materials are summarized in Table 1.

Table 1 Polyelectrolyte characteristics

\begin{tabular}{llll}
\hline Polyelectrolyte & Abbreviation & Type & Molar mass $(\mathrm{g} / \mathrm{mol})$ \\
\hline $\begin{array}{l}\text { Sodium alginate } \\
\text { Poly(L-lysine) } \\
\text { hydrobromide }\end{array}$ & SA & Polyanion & $204,000^{\mathrm{a}}$ \\
$\begin{array}{c}\text { Poly(L-lysine) } \\
\text { hydrobromide }\end{array}$ & PLL2 & Polycation & $27,400-30,200^{\mathrm{b}}$ \\
\begin{tabular}{c} 
Chitosan \\
\hline
\end{tabular} & Ch & Polycation & $2,300-2,900^{\mathrm{b}}$ \\
\hline
\end{tabular}

${ }^{a}$ Calculated from analytical ultracentrifuge sedimentation velocity experiments

${ }^{\mathrm{b}}$ Provided by the supplier

${ }^{c}$ From gel permeation chromatography characterization, polydispersity 1.7
The additives $\mathrm{NaCl}$ and $\mathrm{CaCl}_{2}$ were provided by Fluka (Switzerland).

\section{Method}

All experiments were carried out in an Optima XL-I AUC (Beckman, Palo Alto, CA, USA). The two integrated detection systems, scanning UV/vis, and Rayleigh interference optics [14] served to follow the membrane formation on-line. The principle of the experiment is illustrated in Fig. 1 but has been described, in detail, in preceding publications $[6,15]$.

A systematic study was performed with alginate as a PA and Ch or PLL as a PC (Table 2). The less viscous

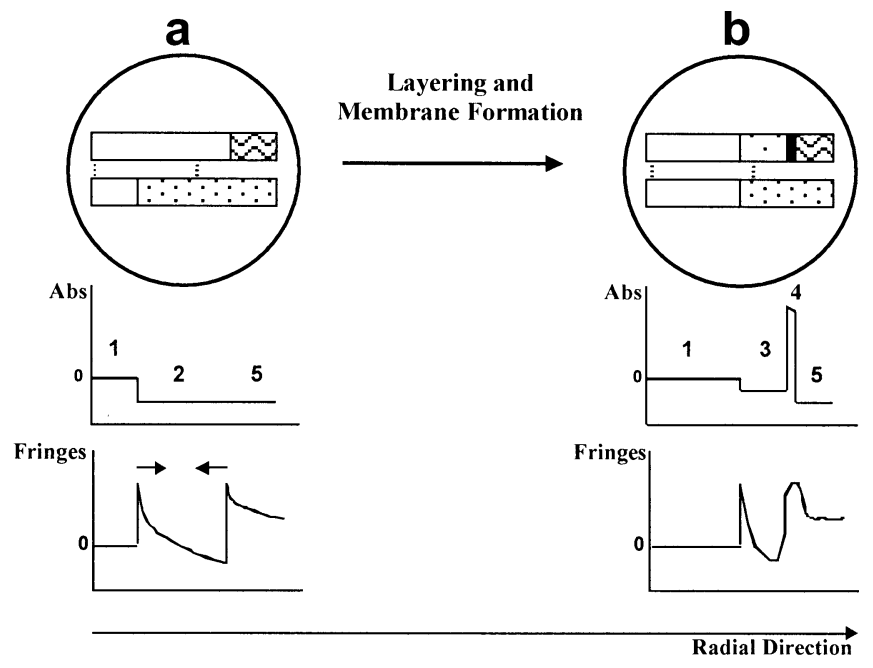

Fig. 1 Principle of the membrane formation experiment in a synthetic boundary cell. The position of the components, the absorption and interference scans are shown, schematically before layering $(a)$ and after layering is complete, though the layered polyelectrolyte is not yet totally consumed for the membrane formation $(b)$. Before layering $(a)$ : the two polyelectrolyte solutions are separated in the two sectors of the double sector cell. Solution 1 (sector with dots), in the lower sector, will pass the capillarylayering solution 2 (sector with wavy lines), in the upper sector, which provides the oppositely charged polyion at its surface. The layering is completed $(b)$ : the solution of the lower sector passes the capillary until reaching the same level in the reaction (upper) sector. It contains solution 2 (sector with wavy lines) with nonreacted polyions, the polyelectrolyte complex (black sector), and solution 1 (sector with dots) with nonreacted polyions. The schematic absorbance scans monitor five scan ranges. These are air/air (1), air/polyelectrolyte solution in the solvent sector (2), polyelectrolyte solution which passed the capillary/polyelectrolyte solution in the solvent sector (3), polyelectrolyte complex membrane/polyelectrolyte in the solvent sector (4), and polyelectrolyte solution in the solution sector/polyelectrolyte solution in the solvent sector (5). The interference scans (fringes as a function of the radial direction) monitor the position of the menisci and their direction of their movement (arrows). The two menisci are superimposed in $b$. The polyelectrolyte complex appears as a nonspecific signal with contributions from the polyelectrolyte network and the salt liberated during the complex formation 
Table 2 Composition of the polyanion and polycation solutions of each experiment selected for the discussion. See Table 1 for abbreviations

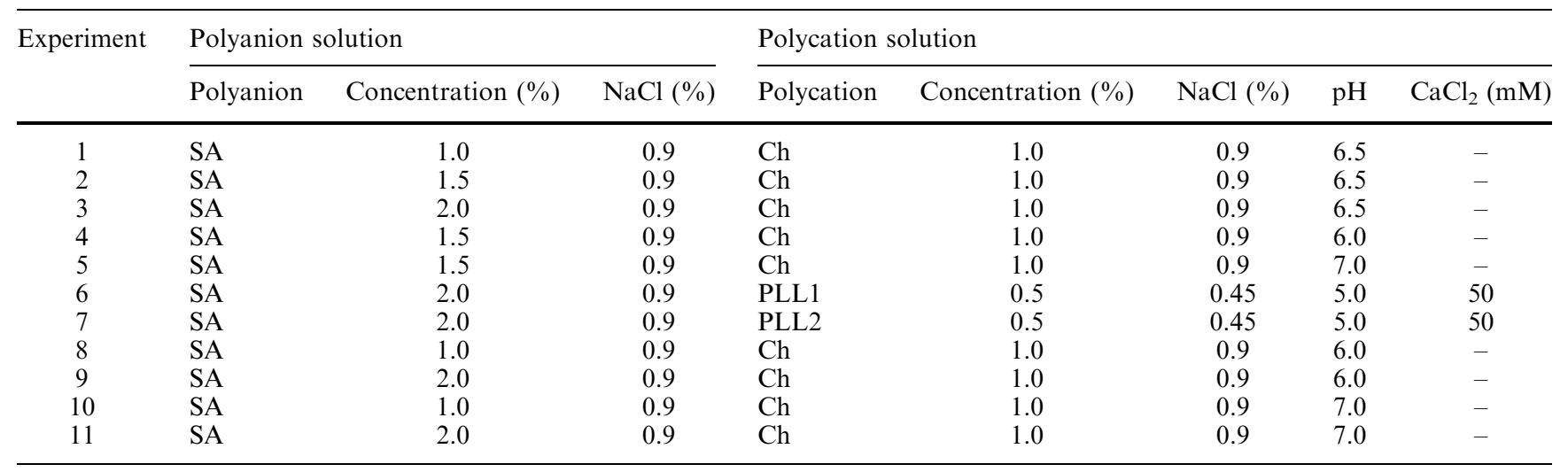

PC solution possessing, in addition, a lower density was filled into the solvent sector and layered during slight acceleration onto the more viscous PA solution in the solution sector. All experiments were performed at $5,000 \mathrm{rpm}$ after $2-3 \mathrm{~min}$ at $1,000 \mathrm{rpm}$. During this short period no layering occurred but the PA solution was removed from the optical lenses. Running at 5,000 rpm the PA solution meniscus becomes sufficiently flat in order not to disturb the detection of the interfacial reaction [6]. However, no sedimentation of the polymeric components occurs at this sedimentation force. The absorbance scans were taken at a wavelength of $370 \mathrm{~nm}$ with a step width of $10 \mu \mathrm{m}$ in a radial direction. Thirty absorbance scans were collected, scans 1-20 with a delay of $2 \mathrm{~min}$, and the following ten scans with a delay of $10 \mathrm{~min}$. The minimum delay depends on the step width and the size of the radial range. The filling volumes were $100 \mu \mathrm{l}$ in the solution sector (PA) and $400 \mu \mathrm{l}$ in the solvent sector (PC). Each experiment was carried out at least in duplicate to ensure reproducibility. The membrane formation was monitored by absorbance scans, whereas interference scans allowed us to calculate the layering velocity from the movement of the meniscus in both the solvent and the solution sector [6].

Note that membrane components with origin from one solution, which participate in the network formation, will be separated by a slash (/) in the text. The components of different solutions will be separated by a double slash $(/ /)$. Application of this formalism to the experiments of this study yields $\mathrm{SA} / / \mathrm{Ch}$ and $\mathrm{SA} / / \mathrm{PLL} / \mathrm{CaCl}_{2}$.

\section{Results}

Two types of raw data were collected during the experiments. First, absorbance scans detecting the membrane formation as a function of time. The superposition of such a series of absorbance scans provides basic information on the network formation process and the geometry of the membrane. Second, the interference scans indicate the position of the meniscus in each of the two sectors. This allows us to know precisely the amount of each polyelectrolyte solution in the two sectors moving into the bottom direction in the solvent sector and in the opposite direction in the solution sector where the reaction occurs (Fig. 1). Since the dimensions of the sectors are known exactly [16] these positions may serve for a precise calculation of the amount of solution in the two sectors.

The six plots in Fig. 2 represent the superposition of the scans collected during the membrane formation of the two-component system SA//Ch. The three plots in the left column correspond to three different SA concentrations, $1.0 \%, 1.5 \%$, and $2.0 \%$ at constant $\mathrm{pH} 6.5$, whereas for the three plots in the right column the $\mathrm{pH}$ was varied in the range 6.0-7.0 keeping constant the SA concentration of $1.5 \%$. From previous qualitative screening experiments an influence on the formation process and the membrane dimensions was expected [7]. Indeed differences are clearly visible for all the plots in Fig. 2. These differences concern the peak position, the peak area, the peak width, and the peak symmetry.

The results of the second experimental series employing PLL of different molar masses as the PC are presented in Fig. 3. The network obtained from the three-component system $\mathrm{SA} / / \mathrm{PLL} / \mathrm{CaCl}_{2}$ is shown there in more detail. $\mathrm{CaCl}_{2}$ was added to PLL to form a gel with SA supporting the very fragile SA//PLL hydrogel network [17]. The calcium ions diffuse very fast into the SA solution but only partially replace the sodium counterions in the alginate used in this study. The scans reveal different membrane dimensions and formation characteristics, which will be discussed in the subsequent paragraphs.

\section{Discussion}

Considering the dimensions of the centerpiece sectors as well as the position of the cell in the rotor of the AUC the $100 \mu \mathrm{PA}$ solution in the solution sector ranges from 

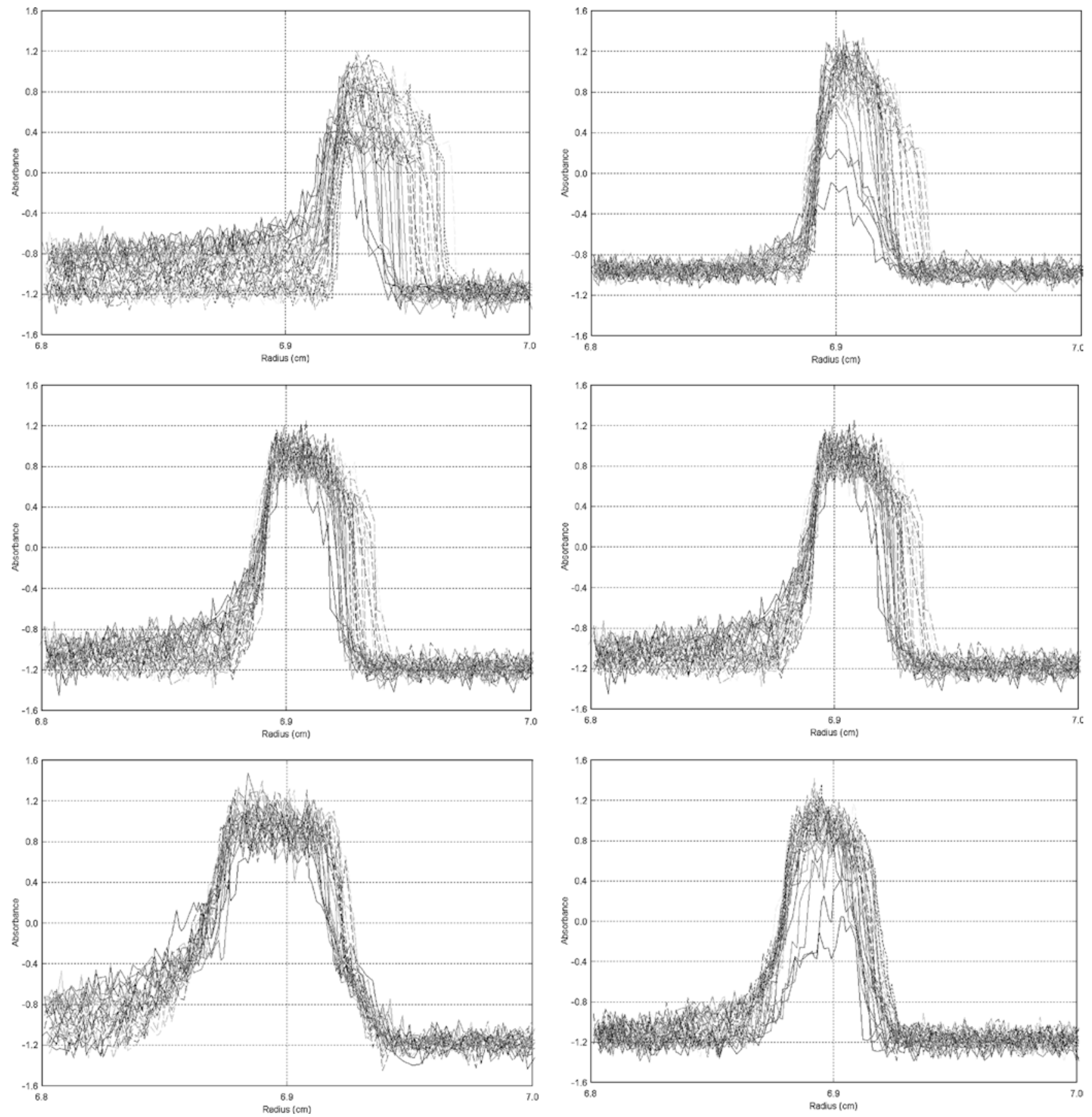

Fig. 2 Analytical ultracentrifuge $(A U C)$ absorbance scans at $370 \mathrm{~nm}$ for sodium alginate layered with chitosan demonstrating the influence of the concentration (three left scans), and the $\mathrm{pH}$ (three right scans) on the polyelectrolyte network formation. Left from top to bottom: experiments 1-3 in Table 2. Right from top to bottom: experiments $4,2,5$ in Table $2.5,000 \mathrm{rpm}, 20{ }^{\circ} \mathrm{C}, 30$ scans, scan delay 2 min for the 20 first scans, 10 min for the last ten scans

6.92 to $7.2 \mathrm{~cm}$ of the radial position before the experiment has started. Consequently, the first contact with the PC solution occurs at $6.92 \mathrm{~cm}$. The scans in Fig. 2

cover the radial range from 6.8 to $7.0 \mathrm{~cm}$. This range was limited to $7.0 \mathrm{~cm}$ and was not extended to the bottom of the cell since the absorbance intensity did not change between 7.0 and $7.2 \mathrm{~cm}$ containing the PA solution. In addition, recording with a short scan delay requires a short radial distance for the selected step width of $10 \mu \mathrm{m}$. The negative intensity of the base lines on both sides of the membrane peak results from detecting the difference between the absorbance in the 
Fig. 3 AUC absorbance scans at $370 \mathrm{~nm}$ for sodium alginate layered with poly(L-lysine) $(P L L) / \mathrm{CaCl}_{2}$ demonstrating the influence of the molar mass of PLL Top: experiment 6 in Table 2. Bottom: experiment 7 in Table 2. 5,000 rpm, $20{ }^{\circ} \mathrm{C}$, 30 scans, scan delay 2 min for the 20 first scans, 10 min for the last ten scans
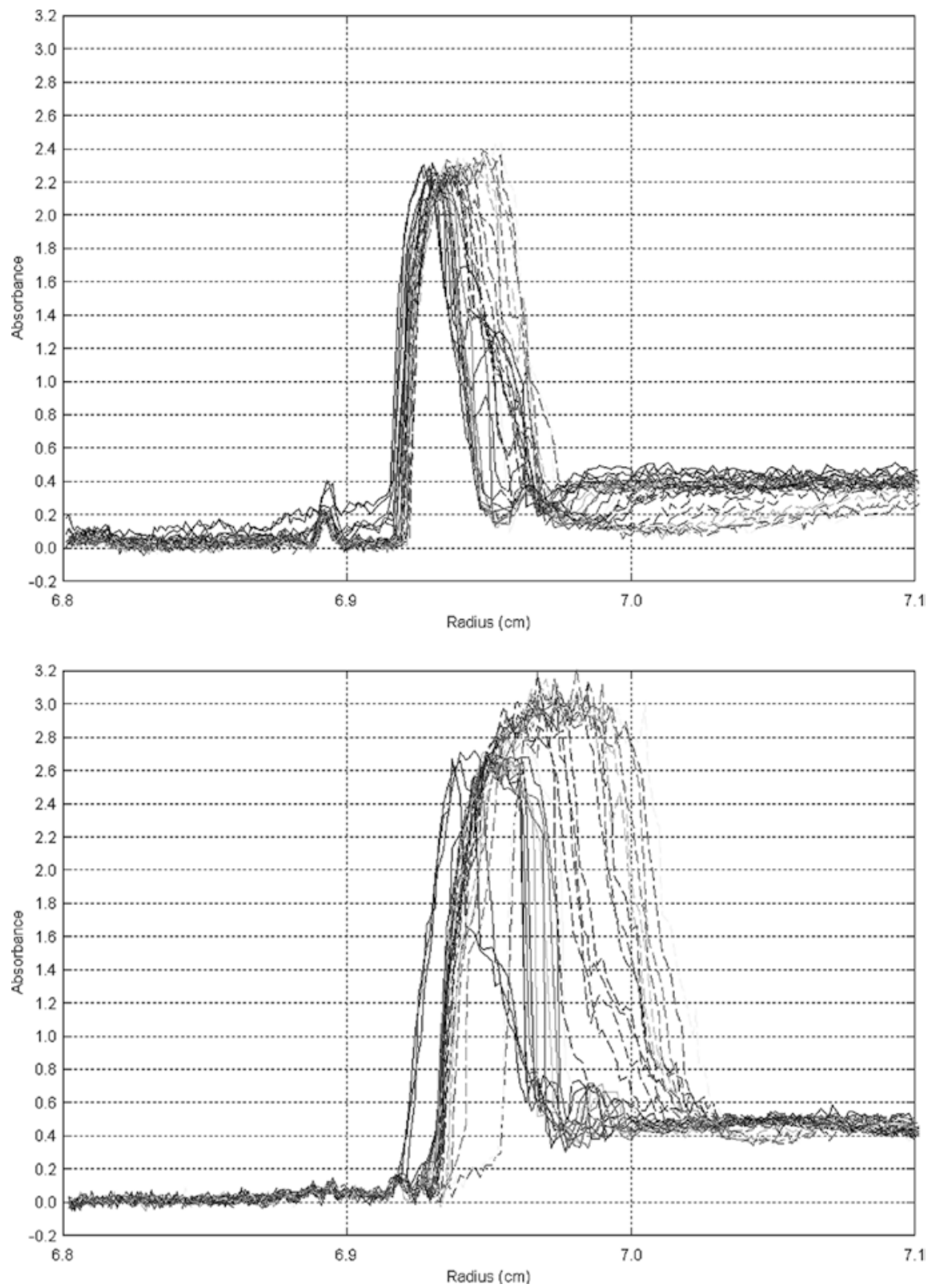

solution and solvent sector in all AUC experiments [8]. The PC sector containing the oligochitosan solutions of the initial concentration in this series of experiments absorbs more strongly than the solutions on both sides of the membrane in the PA sector but not the membrane itself. The decreasing intensity on the left side of the membrane corresponds to the $\mathrm{Ch}$ consumption with time. The constant absorbance on the right side confirms that SA does not absorb, no oligochitosan permeates without complex formation, and no sedimentation takes place. Only for the experiment at $\mathrm{pH} 6.0$ (upper plot of the right column in Fig. 2) does the absorbance have a slightly different level. It is known that the solubility of $\mathrm{Ch}$ improves with decreasing $\mathrm{pH}$; therefore, a better solution state can be suggested at $\mathrm{pH} 6.0$ and, as a consequence, there is less absorbance, which reduces the difference recorded.

The general scan characteristics are different for the second membrane type, SA//PLL $/ \mathrm{CaCl}_{2}$, studied here (Fig. 3). In this case the range between 6.8 and $7.1 \mathrm{~cm}$ was selected for scanning. For this system, both the PA (SA) and the PC (PLL) do not absorb at $370 \mathrm{~nm}$; therefore, the base line appears at zero absorbance on the left side of the membrane peak. The signal on the right side is caused by the turbidity of the supporting calcium alginate gel, which is built immediately and much faster than the polyelectrolyte network, though the membrane range is clearly separated from the 
calcium gel. Regarding the right side of the membrane, in the upper graph, a flat sedimentation velocity profile seems to result. This is explained later in the section Membrane compactness.

The scans contain different information; however, the software package supplied with the AUC does not provide any software for a specific evaluation of this interfacial reaction. For a more detailed interpretation, a procedure has been developed based on MATLAB software, version 6.1.0. Five characteristics have been selected and defined for an objective scan evaluation and conclusions thereof applicable to different types of polyelectrolyte networks formed at the flat interface and possessing limited dimensions. These are total membrane thickness $\left(\mathrm{MT}_{0}\right)$, membrane compactness (MC), membrane heterogeneity $(\mathrm{MH})$, membrane symmetry (MS), and membrane growth and kinetics.

These characteristics will be discussed, in detail, for $\mathrm{SA} / / \mathrm{Ch}$ and $\mathrm{SA} / / \mathrm{PLL} / \mathrm{CaCl}_{2}$. For this purpose, membrane scans taken after $140 \mathrm{~min}$ were extracted from Figs. 2 and 3, and are collected in Figs. 4 and 5. They correspond to the membrane structure at this time. The appropriate characteristic values of all experiments are summarized in Table 3.

\section{Total membrane thickness}

The total membrane thickness is measured as the scan width at a given absorbance, for example, at -0.5 absorbance in the case of the $\mathrm{Ch}$ membranes in this experimental series. Performing the comparison at a fixed intensity corresponding to the same network density seems to be more reliable than to take the values at the base line, which is, in some cases, relatively noisy. It is obvious that the membrane thickness strongly depends on the initial concentration of the PA solution. It increases with increasing SA concentration though differently with the $\mathrm{pH}$ variation. Moreover, the $\mathrm{pH}$ does not influence the membrane thickness to such an extent. Fig. 5 clearly reveals the influence of the molar mass of PLL on the total membrane thickness, with the lower molar mass forming thicker membranes. If compared with the Ch membranes ( $2 \%$ SA concentration), they are slightly thicker for PLL in a similar molar mass range.

\section{Membrane compactness}

The membrane compactness parameter provides information on the density of the polyelectrolyte complex network. This compactness is concluded from the maximum value of the absorbance scan. All SA//Ch membranes exhibit a maximum at approximately 2.2 absorbance units. Both $\mathrm{pH}$ and alginate concentration do not systematically influence the compactness of the
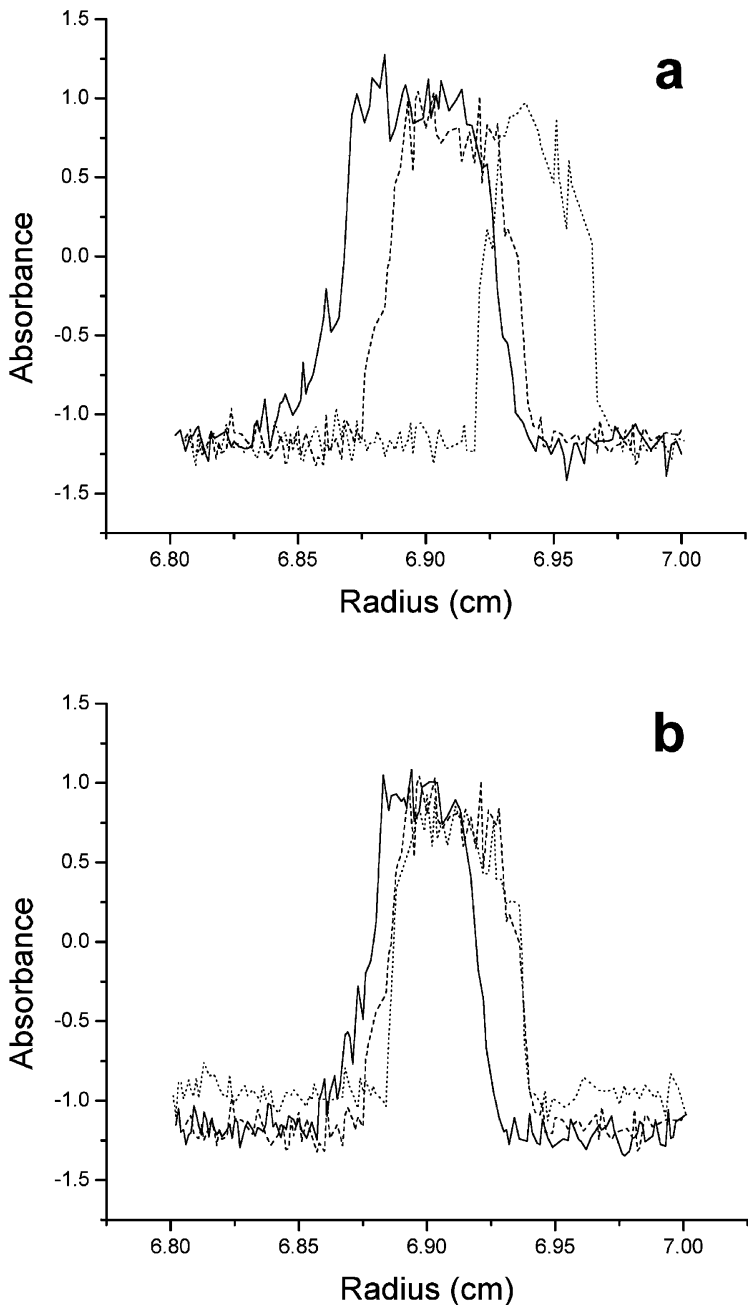

Fig. 4a, b AUC absorbance scans at $370 \mathrm{~nm}$ after 140-min detecting and comparing the membrane characteristics and the membrane position at this time. Scans taken from Fig. 2. a Influence of the concentration: experiment 1 (dotted line), experiment 2 (dashed line), experiment 3 (solid line). b Influence of the $\mathrm{pH}$ : experiment 4 (dotted line), experiment 2 (dashed line), experiment 5 (solid line)

membrane, though a slight decrease may be concluded with decreasing SA concentration. The polyelectrolyte complex network SA//PLL is suggested to be denser in the case of the lower molar mass PLL2, for which a higher absorbance is detected. This suggestion has been verified by permeability measurements clearly indicating a cutoff decrease (denser network) if the membrane SA// PLL was formed with lower molar mass PLL [18]. A further conclusion is that for the thinner and less compact membrane formed with PLL1 less alginate from the PA solution has reacted with PLL1. Consequently, more alginate remained available to react with calcium, resulting in a higher calcium alginate concentration. This calcium alginate could then start to precipitate, causing scans with sedimentation profiles as seem to be 


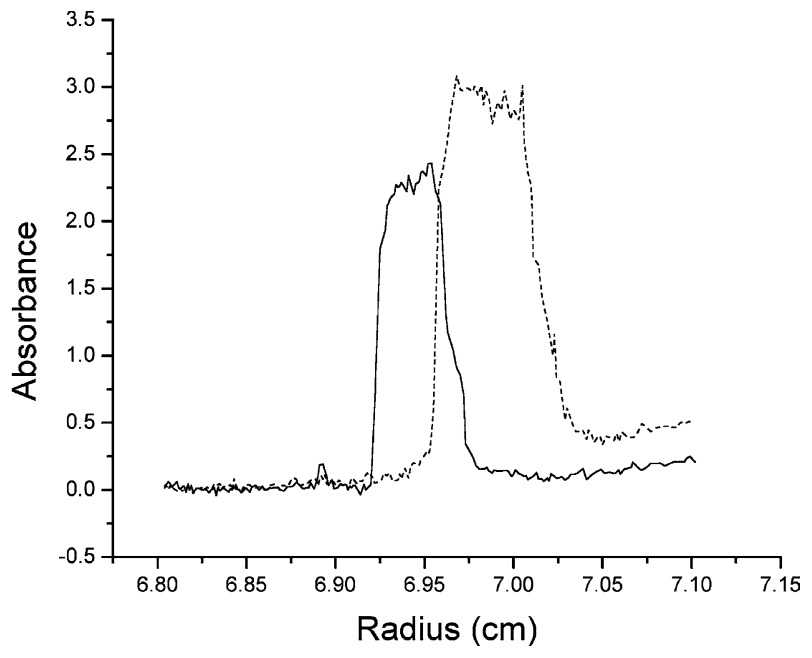

Fig. 5 AUC absorbance scans at $370 \mathrm{~nm}$ after 140-min detecting and comparing the membrane characteristics and the membrane position at this time. Scans taken from Fig. 4. Experiment 6 (solid line), experiment 7 (dashed line)

Table 3 Membrane evaluation. All values are averages of experiments performed, as least, in duplicate

\begin{tabular}{cccc}
\hline Experiment & $\begin{array}{l}\text { Membrane thickness } \\
\mathrm{MT}_{0}(\mu \mathrm{m}) \text { (average) }\end{array}$ & $\begin{array}{l}\text { Heterogeneity } \\
\mathrm{MH} \text { (average) }\end{array}$ & $\begin{array}{l}\text { Symmetry } \\
\text { MS (average) }\end{array}$ \\
\hline 1 & 460 & 0.35 & 0.33 \\
2 & 560 & 0.22 & 0.48 \\
3 & 650 & 0.14 & 0.80 \\
4 & 525 & 0.32 & 0.28 \\
5 & 550 & 0.34 & 0.90 \\
6 & 500 & 0.40 & - \\
7 & 710 & 0.39 & - \\
8 & 620 & 0.30 & 0.60 \\
9 & 680 & 0.24 & 0.80 \\
10 & 540 & 0.22 & 0.50 \\
11 & 890 & 0.38 & 0.91 \\
\hline
\end{tabular}

visible in the upper graph of Fig. 3 on the right side of the membrane peak. The calcium alginate concentration in the lower graph of Fig. 3 is expected to be lower since more alginate is bound to PLL2.

Nevertheless, the impact of several influences on the absorbance signal of the membranes must be carefully distinguished. Clearly, self-absorbing PAs and PCs will contribute. Depending on their concentration, which is expected to be higher in the polyelectrolyte complex, the intensity will increase in comparison to the solution. Furthermore, since the ability of the PA/PC pairs to form polyelectrolyte complex hydrogels and not solid precipitates is determined, to a large extent, by the hydrophile of the polymer backbone, the degree of swelling of the complex affects the signal by more or less pronounced turbidity. In the case of SA//Ch, oligochitosan became absorbing after chain degradation. The absorbance of a $1 \% \mathrm{Ch}$ solution is 1.2 at $370 \mathrm{~nm}$ detected by a

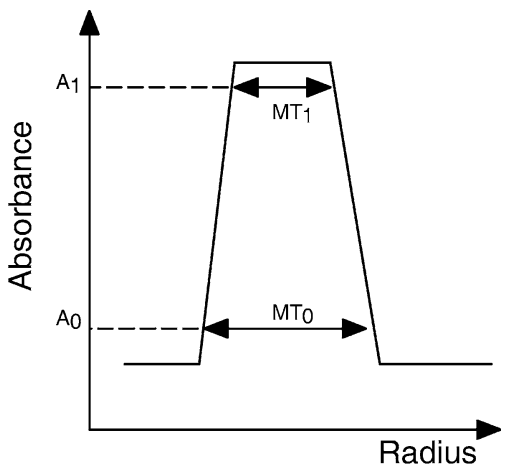

Fig. 6 Principle of the heterogeneity calculation. Total membrane thickness at $A_{0}\left(M T_{0}\right)$, membrane thickness at a second absorbance value $A_{1}\left(M T_{1}\right)$

-1.2 signal in the solution difference scans in the scan plots. The higher membrane signal may, therefore, result from a higher Ch concentration in the membrane and/or a turbidity contribution. As the second system a combination of nonabsorbing components (SA, PLL, $\mathrm{CaCl}_{2}$ ) was selected, with the membrane peak then only related to the turbidity. Considering all these aspects, a quantitative comparison of the compactness between different membrane chemistries needs further investigation.

\section{Membrane heterogeneity}

The heterogeneity of the membrane is defined as the difference between the total membrane thickness $\left(\mathrm{MT}_{0}\right)$, in general taken close to the base line, and the scan width at a second selected absorbance value $\left(\mathrm{MT}_{1}\right)$ related to both the absorbance difference, $A_{1}-A_{0}$, where the thickness was measured, and the total membrane thickness, $\mathrm{MT}_{0}$ :

$\mathrm{MH}=\frac{\mathrm{MT}_{0}-\mathrm{MT}_{1}}{\left(A_{1}-A_{0}\right) \mathrm{MT}_{0}}$.

The heterogeneity becomes zero for $\mathrm{MT}_{0}=\mathrm{MT}_{1}$. This evaluation is presented schematically in Fig. 6. The calculation considers the slope of the scan by relating the scan width difference $\left(\mathrm{MT}_{0}-\mathrm{MT}_{1}\right)$, at two absorbance values, to the appropriate absorbance difference $\left(A_{1}-A_{0}\right)$. This is assumed to correlate to a network density gradient on both sides of the membrane. Since the same slope will be more significant for a thinner membrane than for a thicker one, this ratio is, in addition, related to the total membrane thickness. Such an evaluation provides more information than simply relating the peak area to the width at the maximum of the absorbance value. In the case of $\mathrm{SA} / / \mathrm{Ch}$ the heterogeneity was calculated for the scan widths at -0.5 and 0.5 absorbance. For SA//PLL/ the values in Table 3 relate to $\mathrm{MT}_{0}$ at 1.0 absorbance and $\mathrm{MT}_{1}$ at 2.0 absor- 
bance. Unfortunately, the heterogeneity parameter does not provide information on which side of the membrane the heterogeneity occurs. This was reason for introducing a further parameter, the symmetry parameter.

\section{Membrane symmetry}

The membrane symmetry parameter provides, to a certain extent, information about the diffusion process. Working at low SA concentration, the SA//Ch membrane exhibits a significant absorbance difference on both sides of the membrane peak with a lower one in the SA solution direction. A quantitative, and objective, evaluation of this parameter seems to be more complicated and needs further investigation concerning its generalization.

The membrane peak consists, in some experiments, of two parts: a more regular, more or less heterogeneous one in the lower peak area and a nonsymmetric one on the top. For comprehensive evaluation these two peak parts have to be considered. Nevertheless, real experimental values become available by the AUC method. This is not easy, for example, for imaging methods. As the simplest evaluation for the SA/Ch-type membranes the difference in the absorbance units on both peak sides, $S_{1}$ and $S_{2}$, related to the higher absorbance is proposed:

$\mathrm{MS}=1-\frac{S_{1}-S_{2}}{S_{1}}$,

with $S_{1}=A_{1}-A_{0}$ and $S_{2}=A_{2}-A_{0}$. MS $=1$ for $S_{1}=S_{2}$, a fully symmetric membrane. The estimated symmetries are summarized in the last column of Table 3. A more symmetric membrane is concluded from the scan observation at high alginate concentration $(2 \%$, experiments $3,9,11)$. Keeping the alginate concentration constant, at $1.5 \%$, the symmetry increases with the $\mathrm{pH}$.

The molar masses of PLL have a significant impact on the membrane symmetry. The two membranes in Fig. 5, however, possess a different shape compared with the SA//Ch membranes in Fig. 4. The symmetry for Ch corresponds to an absorbance decrease at the top of the peak. The peak shape for PLL is characterized by the onset of an absorbance decrease in the middle of the peak. Equation (2) is not applicable in this case. The two symmetry types are presented schematically in Fig. 7.

Several mechanisms for the formation of polyelectrolyte complex networks/gels/membranes are under discussion [19] or can be concluded based on the thermodynamics of the process, which may lead to various symmetry and membrane growth processes. Overall, the initial contact of the oppositely charges polyions can result in the formation of a permanent network which is completely neutral and its density allows penetration of later incoming polyions, increasing the membrane thickness. However, there is evidence that the complex underlies dynamics and the polyion chains arriving at the interface replace the network contacts, pushing the polyions of the same charge in the direction of the oppositely charged polyions, which have not reacted yet. Moreover, such a process will be thermodynamically favorable for polydisperse polyion components with the longer chains replacing the shorter ones. On the other hand, the diffusion of shorter chains is favored. But it is also known that shorter chains, in general, form networks possessing lower mechanical stability at comparable concentrations [20].

The different types of peak symmetry for Ch and PLL correspond, for most of the $\mathrm{SA} / \mathrm{Ch}$ experiments, to membranes with a continuously decreasing compactness but still a relatively high compactness in the direction of the SA solution. In contrast, the SA//PLL membranes consist of one relatively homogeneous, symmetric and compact part, which continues in a region of relatively low compactness and, finally, the calcium gel.

\section{Membrane growth and kinetics}

Differences of the membrane formation process are obvious from Figs. 2 and 3. This concerns both the formation of a certain shape and the kinetics. Since for the study of SA//Ch the same components were employed for all experiments the differences result from the variation of the experimental conditions. Probably, lower SA concentrations yield, initially, less dense networks allowing the penetration of further $\mathrm{Ch}$ chains into the less concentrated SA solution. The excess of SA in the case of higher SA concentrations will push the SA chains to react with $\mathrm{Ch}$. This process is indicated by the different positions of the membrane peak in the AUC cell. At low $\mathrm{pH}$, where $\mathrm{Ch}$ is more positively charged, the movement in the SA direction is favored.

The formation process is quantitatively described in Fig. 8, where the increase of the membrane thickness with time is compared for $1 \%$ and $2 \% \mathrm{SA}$ at $\mathrm{pH} 6.5$. At
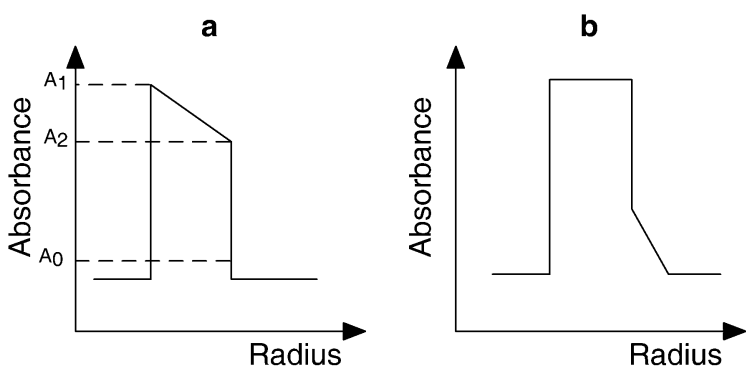

Fig. 7 Schematic presentation of the two membrane symmetry types: a corresponds to sodium alginate//chitosan; $\mathbf{b}$ corresponds to sodium alginate $/ / \mathrm{PLL} / \mathrm{CaCl}_{2}$. Principle of the symmetry calculation in $\mathbf{a}$ 
$1 \%$, the membrane thickness continuously increases, whereas at $2 \%$ SA a final thickness seems to be reached after approximately $50 \mathrm{~min}$. Clearly, this process is also influenced by the availability of $\mathrm{Ch}$, which, indeed, was kept constant for the two experiments.

If not only the increase of the membrane thickness as a function of time is plotted but also the change of the radial positions at selected absorbance with time, as presented in Fig. 9 for SA/PLL, a more comprehensive description of the growth process becomes accessible. It analyzes the movement of the contact interface, the growth direction, and may serve to calculate the growth velocity from the slop. A much faster growth is visible for PLL2. Moreover, if there is a minor difference in the radial positions at various absorbance values a more homogeneous network with well-defined surfaces can be concluded. The difference of the upper and lower curves corresponds, for the same absorbance, to the scan width at this position. A quantification of the formation kinetics has to consider several steps by different mechanisms: spontaneous network formation when the first contact of the PA and PC chains takes place, a very fast process; diffusion and/or movement of the polyions influenced, for example, by the characteristics of the initially formed network, the macromolecular and electrochemical characteristics of the components, or medium conditions, a process of moderate velocity; network equilibration and/or reorganization, a slow to very slow process [6].

\section{Further characteristics}

The absorbance scans, but also the interference scans, provide further information, which is not easy to generalize. As has already been mentioned, the same

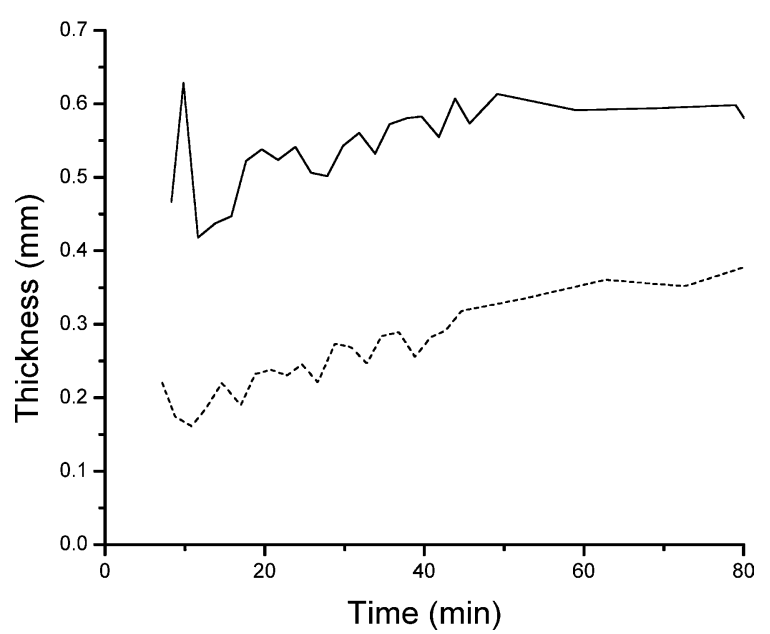

Fig. 8 Membrane thickness as a function of time. Experiment 1 (dashed line), experiment 3 (solid line) amount of SA solution, $100 \mu \mathrm{l}$, was always filled into the AUC cell. The various positions of the membrane peaks deserve, therefore, attention. They may result from the formation mechanism influenced by the experimental conditions. In addition, shrinking during complex formation and/or osmotic influences can superimpose the detection of the formation process. The position of the peak, in the case of the SA concentration variation, correlates with the interpretation that, at low concentration, the diffusion of $\mathrm{Ch}$ through a then less dense network is favored. At high SA concentration the SA replacement in the network may serve as an explanation. At low $\mathrm{pH}$ the electrostatic attraction of the more positively charged $\mathrm{Ch}$ may cause the position. At higher $\mathrm{pH}$ this effect is less pronounced. The position of the peak may be useful to support a mechanistic explanation. In the case of PLL the lower molar mass PLL2 will diffuse faster into the SA solution. The thicker and more compact membrane probably causes strong shrinking.
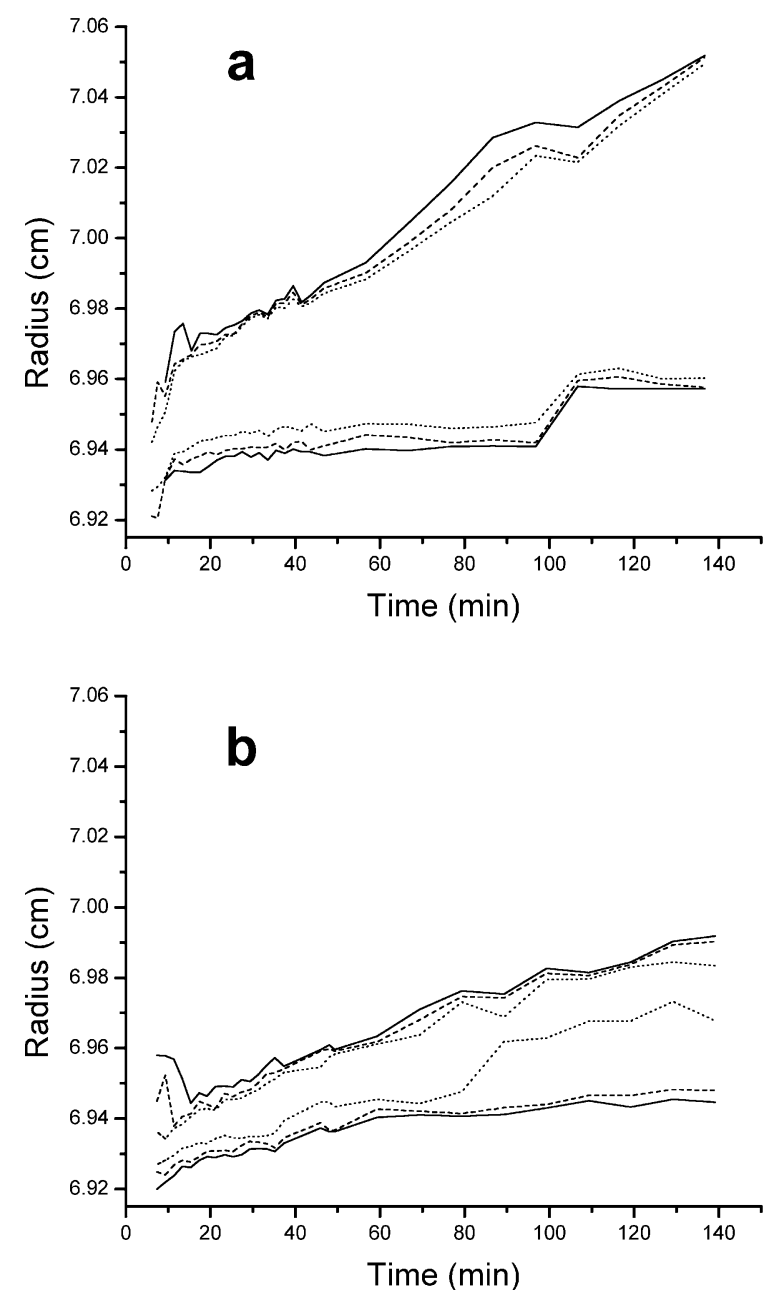

Fig. 9a, b Membrane growth as a function of time at various absorbances: 1.0 (solid line), 1.5 (dashed line), 2.0 (dotted line). a Experiment 7, b experiment 8 


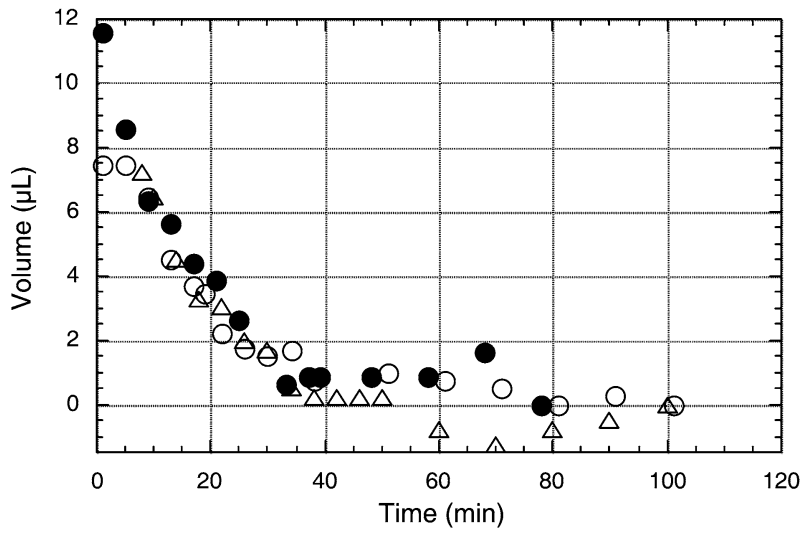

Fig. 10 Volume difference during the complex formation as a function of time (volume decrease in the polycation sector minus volume increase in the polyanion sector). Experiment 8 (closed circles), experiment 4 (open circles), experiment 9 (triangles)

The total membrane thickness, $\mathrm{MT}_{0}$, was estimated somewhat far from the base line. In particular, in the case of SA//Ch, at $2 \% \mathrm{SA}$, a peak broadening at low absorbance appears. This corresponds to a less defined and rough surface of the membrane. A well-defined and steep absorbance jump refers to a flat, sharp surface. In general, the surfaces of the PLL membranes are better defined than the SA//Ch surfaces.

The interference scans serve to calculate the amount of solvent in the two sectors. In detail, from the movement of the meniscus in the PC sector the volume decrease is available. The movement of the meniscus in the PA sector reflects the volume increase there. Both should be identical, but were not for these experiments. Figure 10 shows the calculated differences, for three experiments, decreasing with time. This is the difference between the expected volume increase and the real increase, more precisely, the volume in the PA sector increases less than expected from additivity. The volume loss identifies the shrinking during the complex formation, a process that is difficult to follow experimentally but becomes accessible here.

\section{Conclusions and outlook}

The systematic experiments performed clearly demonstrate the influence of the concentration, $\mathrm{pH}$, and molar mass on the polyelectrolyte network formation at a welldefined flat interface. The recently developed AUC technique provides a multitude of information about the formation process and the network characteristics. All the information that can be taken from the AUC scans has been generalized, and exploited, by introducing and defining characteristics which allow the comparison of different membrane types. Although some of them, the membrane compactness, heterogeneity, and symmetry, are qualitative parameters as they depend on the turbidity reading via absorption optics, all characteristics together will contribute to establish qualitative and partly quantitative relationships for the influence of the polyelectrolyte properties and the experimental conditions on the membrane formation process and the membrane properties. Such relationships will be used to support the development of membranes for specific applications. Further investigations are in progress to correlate the experimental findings from AUC with results from other methods such as mechanical stability and permeability. Overall, AUC is a valuable and unique tool to study on-line polyelectrolyte network formation in detail.

Acknowledgements We thank P. Schuck, National Institute of Health, Bethesda, USA, for his support developing the evaluation software. W. Strand, Norwegian University of Science and Technology, Trondheim, Norway, is acknowledged for the alginate structural analysis. Furthermore, the authors gratefully acknowledge financial support by the Swiss National Science Foundation, grant 2100-064996.

\section{References}

1. Dautzenberg H, Jaeger W, Kötz J, Philipp B, Seidel C (1994) Polyelectrolytes: formation, characterization and applications. Hanser, Munich

2. Zintchenko A, Rother G, Dautzenberg H (2003) Langmuir 19:2507

3. Kabanov VA, Zezin AB (1984) Macromol Chem 6:259

4. Dautzenberg H (2001) In: Radeva $T$ (ed) Physical chemistry of polyelectrolytes. Surfactant science series, vol 99. Dekker, New York, p 743
5. Gander B, Blanco-Prieto MJ, Thomasin C, Wandrey C, Hunkeler D (2001) In: Swarbrick J, Boylan JC (eds) Encyclopedia of pharmaceutical technology, 2nd edn. Dekker, New York, p 481

6. Wandrey C, Bartkowiak A (2001) Colloids Surf A 180:141

7. Wandrey C, Grigorescu G, Hunkeler D (2002) Prog Colloid Polym Sci 119:84

8. Cölfen H (1998) Polym News 23:5

9. Cölfen H (1999) In: Harding SE (ed) Biotechnology and genetic engineering reviews. , p 87

10. Börger L, Cölfen H (1999) Prog Colloid Polym Sci 113:23
11. Lucas G, Börger L, Cölfen H (2002) Prog Colloid Polym Sci 119:11

12. Draget KI, Skjåk-Bræk G, Smidsrod O (1994) Carbohydr Polym 25:31

13. Bartkowiak A, Hunkeler D (2000) Chem Mater 12:206

14. Beckman Instruments, Inc (1998) Model XL-I analytical ultracentrifuge. Beckman Instruments, Inc, Palo Alto, USA

15. Wandrey C, Bartkowiak A, Hunkeler D (2000) In: Transact 6th World Biomat Congr 15-20 May, 2000, Hawaii USA, vol II. Soc. Biomat., USA, p 893 
16. Chervenka CH (1969) A manual of methods for the analytical ultracentrifuge. Spinco Dev Instr, Palo Alto 17. Lim, F., Science (1980) 210.
18. Gugerli R (2003) Doctoral thesis no 2720. Swiss Federal Institute of

Technology, Lausanne, Switzerland 19. Potemkin II, Khokhlov AR (2002) In: Osada Y, Khokhlov AR (eds) Polymer gels and networks. Dekker, New York, p 47
20. Wandrey C, Espinosa D, Rehor A, Hunkeler D (2003) J Microencapsulation 20:597 